\title{
Remote Raman sensor system for testing of rocks and minerals
}

\author{
Christopher S. Garcia a, M. Nurul Abedin ${ }^{\mathrm{b}}$, Shiv K. Sharma ${ }^{\mathrm{c}}$, Anupam K. Misra ${ }^{\mathrm{c}}$, \\ Syed Ismail ${ }^{\mathrm{b}}$, Stephen P. Sandford ${ }^{\mathrm{b}}$, and Hani Elsayed-Ali ${ }^{\mathrm{a}}$ \\ aOld Dominion University, Hampton Blvd, Norfolk, VA 23529 \\ ${ }^{b}$ NASA Langley Research Center, Hampton, VA 23681 \\ ${ }^{c}$ Hawaii Institute of Geophysics and Planetology, University of Hawaii \\ 1680 East-West Rd., POST 602, Honolulu, HI 96822
}

\begin{abstract}
Recent and future explorations of Mars and lunar surfaces through rovers and landers have spawned great interest in developing an instrument that can perform in-situ analysis of minerals on planetary surfaces. Several research groups have anticipated that for such analysis, Raman spectroscopy is the best suited technique because it can unambiguously provide the composition and structure of a material. A remote pulsed Raman spectroscopy system for analyzing minerals was demonstrated at NASA Langley Research Center in collaboration with the University of Hawaii. This system utilizes a $532 \mathrm{~nm}$ pulsed laser as an excitation wavelength, and a telescope with a 4-inch aperture for collecting backscattered radiation. A spectrograph equipped with a super notch filter for attenuating Rayleigh scattering is used to analyze the scattered signal. To form the Raman spectrum, the spectrograph utilizes a holographic transmission grating that simultaneously disperses two spectral tracks on the detector for increased spectral range. The spectrum is recorded on an intensified charge-coupled device (ICCD) camera system, which provides high gain to allow detection of inherently weak Stokes lines. To evaluate the performance of the system, Raman standards such as calcite and naphthalene are analyzed. Several sets of rock and gemstone samples obtained from Ward's Natural Science are tested using the Raman spectroscopy system. In addition, Raman spectra of combustible substances such acetone and isopropanol are also obtained. Results obtained from those samples and combustible substances are presented.
\end{abstract}

Keywords: Raman Spectroscopy, pulsed laser, minerals, ICCD, telescope, rocks, gemstone, remote sensing.

\section{INTRODUCTION}

It has been reported and demonstrated in several papers that Raman spectroscopy is the best suited tool for analysis of minerals on planetary surfaces ${ }^{1-6}$ Current remote Raman spectroscopy systems have the capability to perform remote analysis from within a lander or rover. This capability allows both independent investigations of the surface and identification of important rocks and minerals to be collected for more thorough analysis. In addition, Raman spectroscopy of minerals and their mixtures yields sharp spectral features, which allows for much less ambiguous detection compared to other techniques such as IR spectroscopy. Visible, near infrared, thermal, and reflectance and emission spectroscopy of minerals and compounds all suffer from broad overlapping features that complicate interpretation of their spectra. ${ }^{2}$ On the other hand, the Raman spectra of minerals and their mixtures exhibit sharp and largely non-overlapping features that are much more easily identified and assigned to various mineral species.

A portable remote Raman system was developed at Langley Research Center for measuring the Raman spectra of various materials from a distance of the order of tens of meters. In the intended application of this system, measurements will be performed outdoors where the amount of ambient light cannot be controlled. Previously reported remote Raman systems that utilized cw lasers were only capable of collecting data if the background radiation is very low. ${ }^{1,7}$ Therefore, this Raman spectroscopy system is designed to be operated in environments with high background radiation such as a well illuminated room and under daylight conditions by utilizing a pulsed laser and a gated detector. 
Because this remote Raman system is intended for in-situ analysis of planetary surfaces, the system was tested on several rock and mineral samples. The objective of this study is to observe the capability of the system to obtain Raman spectra from a wide range of unprepared rock and mineral samples of various colors, size, shape, and other properties. In addition, we would like to observe the capability of the system to reproduce similar Raman spectra from various samples of a particular mineral.

\section{EXPERIMENTAL SETUP AND PROCEDURE}

The Raman spectroscopy system at Langley Research Center consists mainly of a laser, a telescope, a spectrograph and a camera. The schematic diagram of the system is shown in Figure 1.

The laser, which serves as the excitation source is a mini Nd:YAG pulsed laser from Big Sky Laser (model UltraCFR) with a $532 \mathrm{~nm}$ wavelength, $45 \mathrm{~mJ} /$ pulse total energy, up to $20 \mathrm{~Hz}$ lasing frequency, and $8 \mathrm{~ns}$ pulse width. Several papers have reported that for such remote Raman systems, pulsed laser is more favorable than a cw laser when measurements are to be taken in daytime conditions. ${ }^{2-5}$ The laser beam is made collinear with optical axis of the telescope using two $45^{\circ}$ prisms to achieve a configuration referred to as coaxial geometry. Maximum performance of the system is achieved with this configuration by permitting scattered signal to be gathered from the target for the $180^{\circ}$ backscattering. The laser hits the target sample placed at $5.6 \mathrm{~m}$ from the system.

The light scattered by the sample is collected by a telescope (Meade ETX-105, Maksutov-Cassegrain telescope with a 4-inch aperture). The telescope output is then focused into the spectrograph entrance aperture by placing a $20 \mathrm{X}$ microscope objective in between. Previously reported similar systems utilized a fiber optic cable to couple the telescope with the spectrograph, but proved impractical due to significant signal attenuation in the cable, difficulty in aligning the components involved, and limitation in the resulting sampling depth of the system. ${ }^{3}$

As the scattered radiation enters the spectrograph (Kaiser Optical Systems, Inc., HoloSpec f/1.8i), the signal is collimated and passed through a notch filter (Kaiser Optical $532 \mathrm{~nm}$ Holographic SuperNotch-Plus Filter) to block the strong Rayleigh-scattered photons. The holographic filter allows a strong attenuation of the Rayleigh signal, while permitting a good transmission of the signal outside the rejection band. ${ }^{8}$ It also features a very narrow rejection bandwidth and sharp spectral edges. The remaining signal is then focused onto a $100 \mu \mathrm{m}$ slit to define the resolution of the spectral lines, then collimated and passed though a holographic transmission grating (Kaiser Optical HoloPlex transmission grating) to disperse the signal into its spectral components. An innovative feature of the HoloPlex transmission grating is that it simultaneously disperses two spectral tracks on the detector; the low frequency portion of the spectra is dispersed on the upper half of detector, and the high frequency portion is dispersed on the lower half. ${ }^{9}$ This scheme effectively doubles the spectral range of the spectrograph while maintaining its resolution.

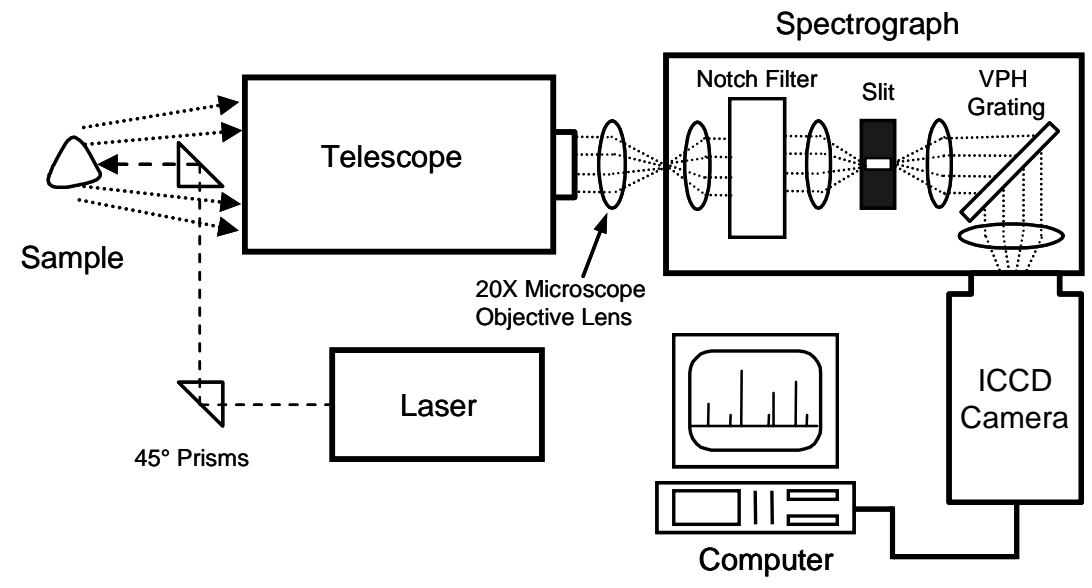

Figure 1. Schematic diagram of the remote pulsed laser Raman spectroscopy system. 
The spectra formed by the spectrograph is measured by a directly coupled thermolelectrically cooled, gated, and intensified charge coupled devices (ICCD) camera (Princeton Instruments PI-Max ICCD camera) with 1024 x 256 pixels and 26 x26 $\mu \mathrm{m}$ pixel size. The ICCD detects extremely weak Stokes lines by adding a gain of up to 250X to the signal. ${ }^{10}$ This is implemented by an intensifier, mainly composed of photocathodes and microchannel plates, placed in front of the CCD. To display, analyze and record the resulting spectra, the CCD is connected to a computer, which runs the CCD application software (Princeton Instruments Winspec/32, Version 2.5F). The spectra were processed and analyzed using GRAMS/AI (Galactic Industries) software.

An important feature of the ICCD camera is its capability for gated operation. This feature is essential in performing experiments in high-background environments such as daylight and room light on. ${ }^{3-6}$ Since the laser only fires an 8ns pulse at a rate of up to 8 pulses per second, and Raman scattering has a relatively very small time constant, the camera can be turned on just right before a laser pulse, and then turned off immediately right after a laser pulse. This should minimize the background radiation that would have been picked up by the camera when the laser pulse is not present.

Several sets of mineral samples were purchased from Ward's Natural Science. All samples were analyzed without cleaning, polishing or any other preparation. They were placed on a stage, which is located about $5.6 \mathrm{~m}$ from the system.

For each sample, the Raman spectrum was collected by firing the laser and synchronously turning the camera on for an equivalent integration time of 10 seconds. The ICCD detector records the "image" of the spectrum, where the horizontal position of the collected charge on a CCD pixel corresponds to the horizontal position of the spectral line and the amount of the charge collected corresponds to its intensity. The resulting spectral image contains both the low frequency range and high frequency range of the spectrum in the top half and the lower half of the CCD array, respectively. The spectral image is then converted into two spectra, the low frequency spectrum and high frequency spectrum, by doing a cross section of the corresponding half of the array.

To convert the horizontal axis of the spectra from pixel position to nanometers and wavenumbers $\left(\mathrm{cm}^{-1}\right)$, the spectral lines of a Neon lamp was measured with the system. The pixel positions of the measured spectral lines were compared to corresponding standard values in nanometers. The performance of the Raman spectroscopy system was evaluated by measuring the spectra of standard samples whose Raman spectra are well known. This procedure is described in detail elsewhere. ${ }^{6}$

\section{RESULTS AND DISCUSSION}

One of the main features of the system is its ability to measure in daylights conditions by gating the ICCD detector. Gating the detector improves the signal-to-background ratio of the Raman peaks. By making the gate width as narrow as possible, the detector picks up less background radiation. Figure 2a and $2 \mathrm{~b}$ shows the spectra of calcite and liquid water, respectively, employing gate widths of $2 \mu$ s and $22 \mathrm{~ns}$. During earlier experiments with this system, a gate width of $2 \mu \mathrm{s}$ was chosen. Although identifiable Raman lines were observed at this gate width as seen in both calcite and water spectra (dashed lines in Figure 2), some of the spectra still exhibit large peaks at the frequency corresponding to the laser signal and high background noise from long-lived fluorescence. Since the laser pulse width is only $8 \mathrm{~ns}$, the detector gate width was further shortened to $22 \mathrm{~ns}$. This gate width was chosen to be more than twice the width of the laser pulse, taking into account the small uncertainty in the actual firing of the laser pulse. From the water spectra shown in Figure 2b, it can be seen that the laser line in the spectrum taken with 22 ns gate width is much weaker than in the spectrum taken with wider gate width. In addition, the spectra of calcite in Figure 2a, the background noise is less in the spectrum taken with the 22 ns gate width. For measurements under room light, this difference may be insignificant especially for materials like calcite, which has strong Raman peaks. However, for outdoor measurements under broad daylight where much higher background radiation is present, this difference is relevant. 


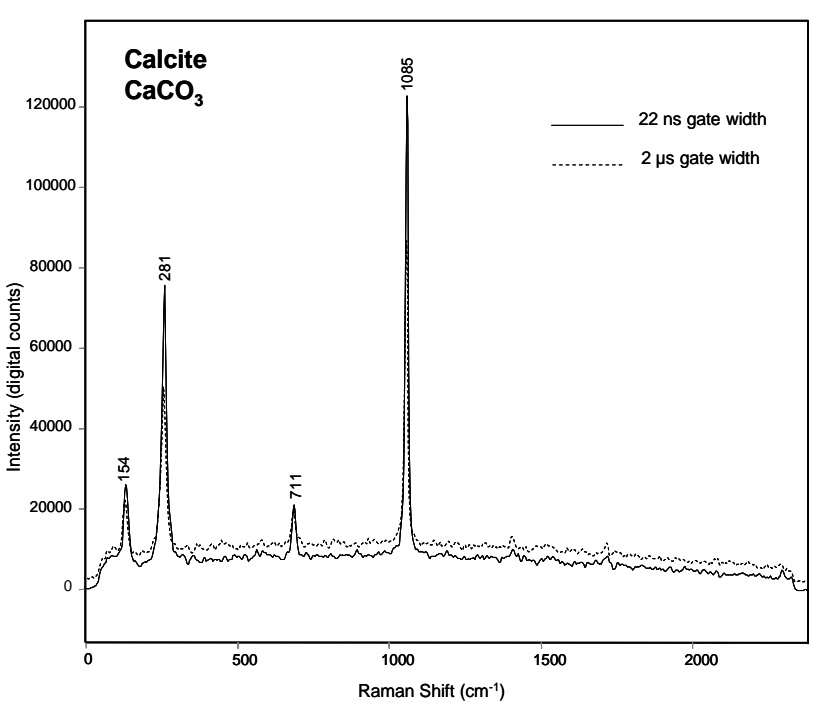

(a)

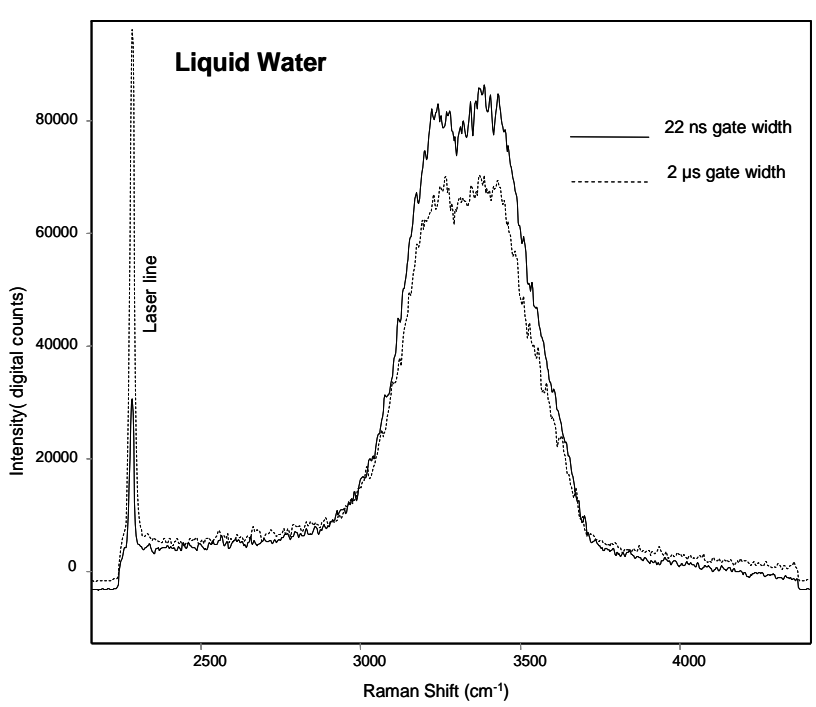

(b)

Figure 2. Raman Spectra of (a) Calcite and (b) liquid water, each showing spectrum obtained using $2 \mu$ and 22 ns gate widths.

The Raman spectra of various samples of calcite and marble are shown in Figure 3a along with the spectra of various quartz samples shown in Figure 3b. These spectra were obtained using an even shorter gate width of $12 \mathrm{~ns}$ and exposure time of $10 \mathrm{~s}$. The first set of spectra in Figure 3a shows the fingerprint peaks of $\mathrm{CaCO}_{3}$ at 1085, 711, 281 and $154 \mathrm{~cm}^{-1}$ in three different calcite samples. The Raman peaks in the marble spectrum in the same locations are characteristic of $\mathrm{CaCO}_{3}$ crystallized in calcite structure. ${ }^{12}$ The strong bands at $1085 \mathrm{~cm}^{-1}$ are due to the symmetric stretching mode of vibration, $v_{1}$ of the carbonate ions $\left(\mathrm{CO}_{3}{ }^{2-}\right)$. The bands at 711 are due to the in-plane bending mode, $v_{4}$ of the carbonate ions and the bands at 154 and $281 \mathrm{~cm}^{-1}$ are due to the low frequency lattice modes of $\mathrm{CaCO}_{3}$ crystallized in calcite structure. ${ }^{12}$ The spectra of calcite samples 2 and 3, show almost no background, whereas the spectrum of calcite sample 1 show significant amount of background. This may be due to the difference in color of the samples. Calcite samples 2 and 3 are very clear and almost transparent, whereas sample 1 is somewhat opaque and much less transparent than the other two samples. Furthermore, calcite samples 2 and 3 show two additional weak bands at 1434 and $1748 \mathrm{~cm}^{-1}$ corresponding to asymmetric stretching mode, $v_{3}$ and combination mode, $v_{1}+v_{4}$, respectively. Marble sample 2, which is a much smaller sample (about $1 \mathrm{~cm}^{3}$ ) compared to marble sample 1 also exhibits high background. The weak bands at 1556 and around $2300 \mathrm{~cm}^{-1}$ that are present in some of the spectra are due to atmospheric $\mathrm{O}_{2}$ and $\mathrm{N}_{2}$, respectively.

In Figure 3b, the quartz samples show characteristic bands at 463 and $205 \mathrm{~cm}^{-1}$, which are due to symmetric stretching of Si-O-Si and lattice distortion, respectively. ${ }^{4}$ The spectra of all the three quartz samples and the rose quartz display the two characteristic bands very well. However, the quartzite sample shows relatively weaker bands. This may be due to the presence of other trace minerals in the sample.

In Figure 4, the Raman spectra of sulfate minerals gypsum $\left(\mathrm{CaSO}_{4} \cdot 2 \mathrm{H}_{2} 0\right)$ and alabaster are shown in both the low frequency range (Figure 5a) and the high frequency range (Figure 5b). These spectra were obtained using 12 ns gate width and an integration time of 10s. All the spectra in the low frequency range in Figure 4a show the strong band at about $1011 \mathrm{~cm}^{-1}$. In addition, the weak bands at around 413, 492, 625, and $1134 \mathrm{~cm}^{-1}$ can be easily identified in all the spectra except in the spectrum of gypsum sample 2. The observed bands are very much comparable to the reported Raman modes for gypsum at 413, 492, 621, 1006, and $1138 \mathrm{~cm}^{-1}{ }^{13}$ In the high frequency spectra shown in Figure 4b, two sharp bands at around 3400 and $3487 \mathrm{~cm}^{-1}$ are observed. These are due to the stretching modes of water molecules, which indicate the presence of chemically bonded two types of water molecules in an ordered structure. ${ }^{14-15}$

The spectra of other minerals that yield identifiable Raman peaks are shown in Figure 5. The Raman spectra of barite, feldspar, granite, fluorite, and steatite were obtained using a gate width of $12 \mathrm{~ns}$ and a 10 s integration time. 


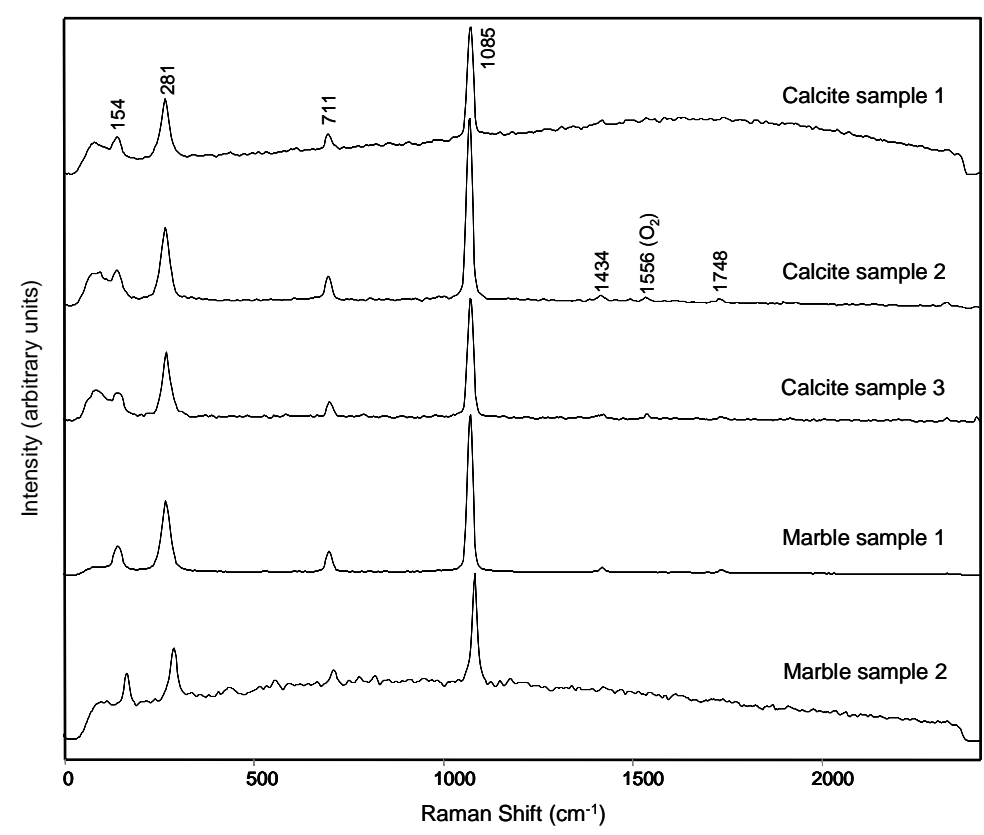

(a)

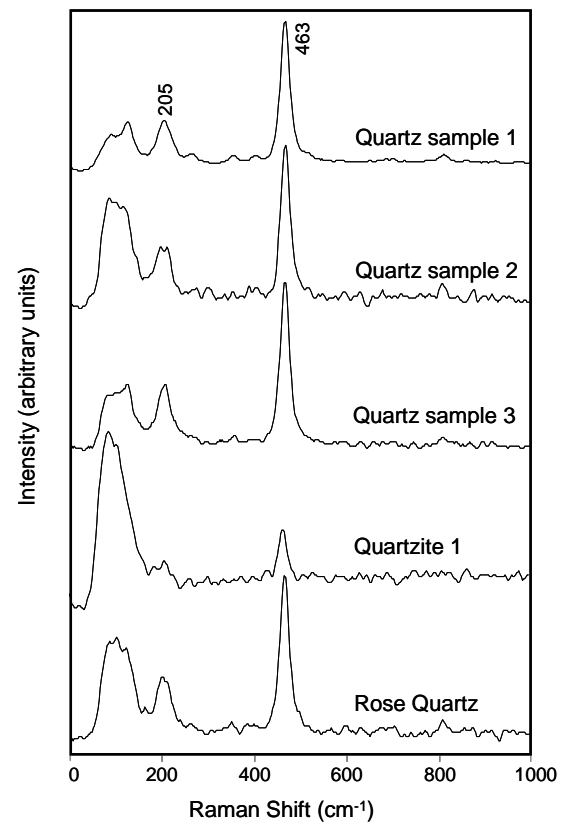

(b)

Figure 3. Comparing Raman spectra obtained from (a) various calcite and marble samples, and (b) various quartz samples.

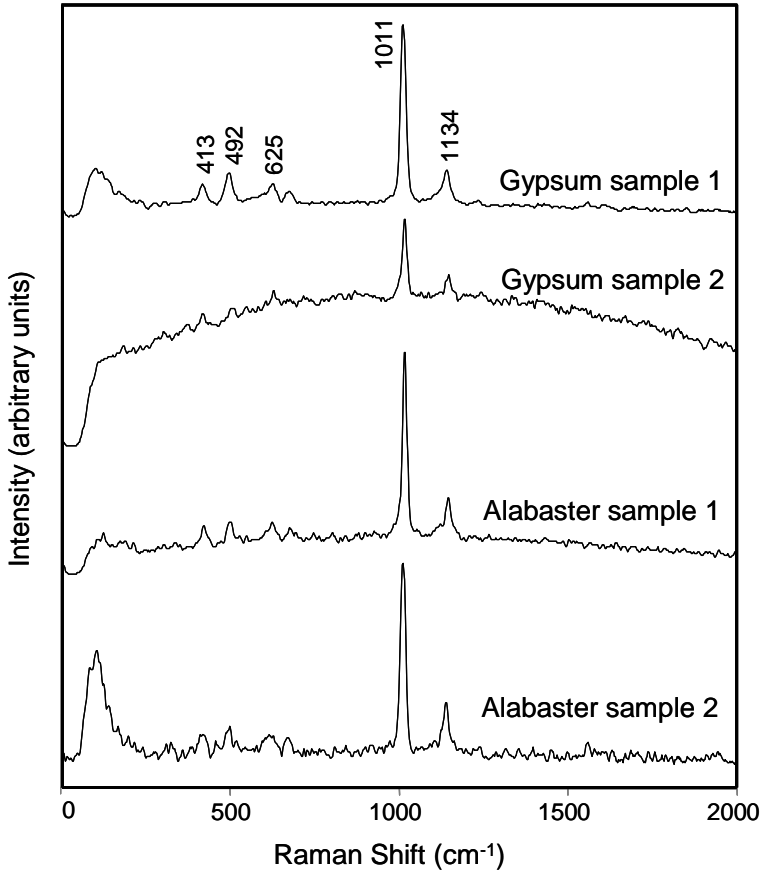

(a)

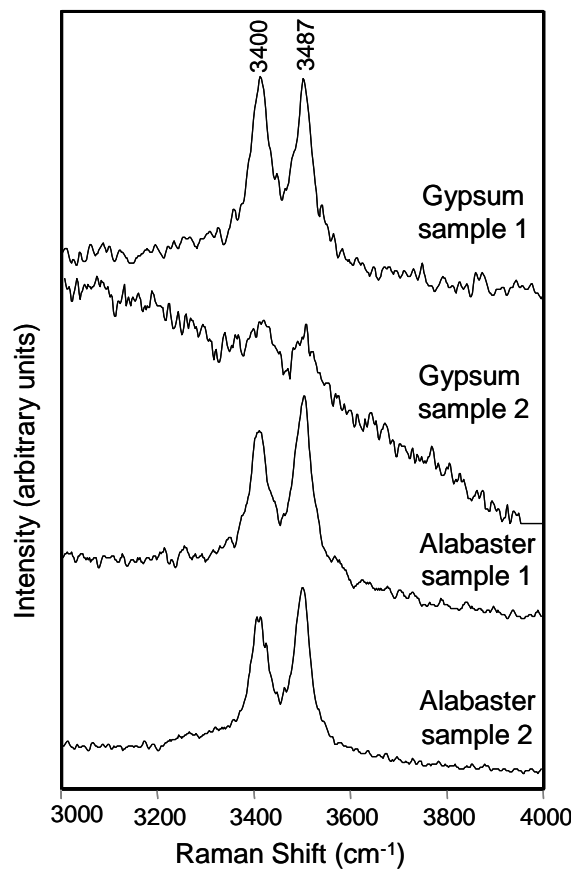

(b)

Figure 4. Comparing Raman spectra obtained from various gypsum samples in the (a) low frequency $\left(0-2000 \mathrm{~cm}^{-1}\right)$ and (b) high frequency (3000-4000 $\mathrm{cm}^{-1}$ ) shift regions. 


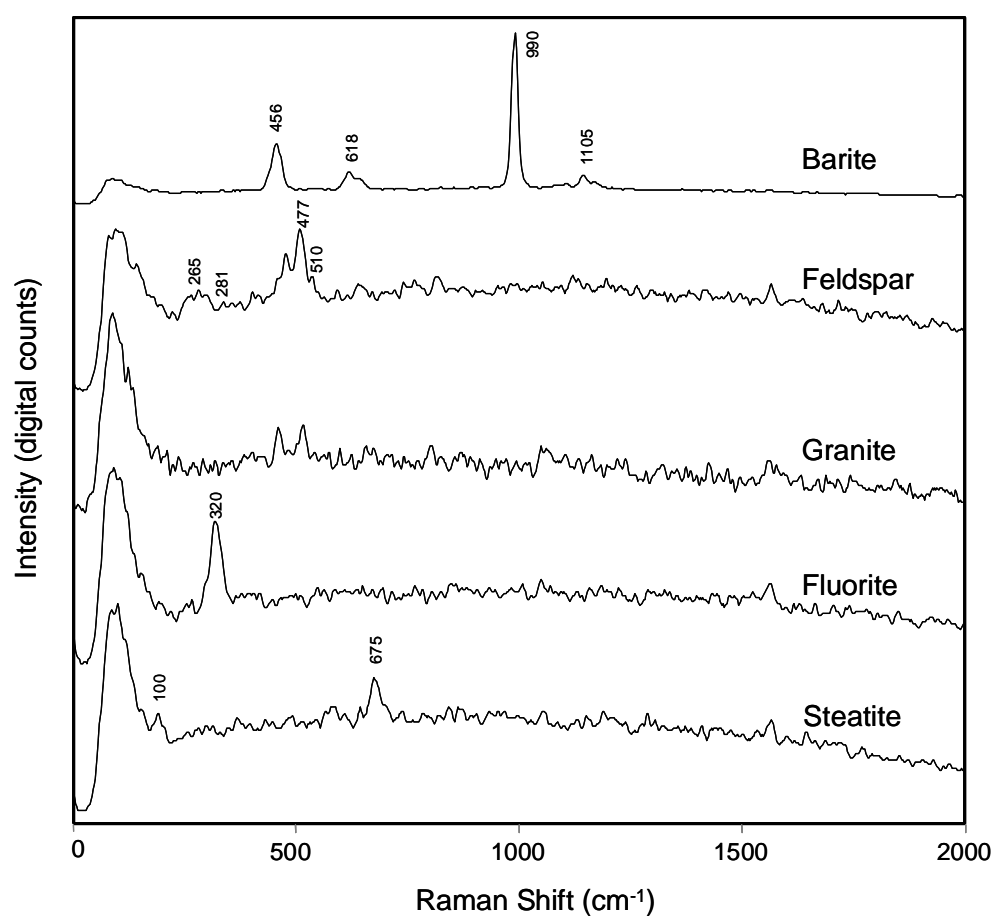

Figure 5. Raman spectra obtained from other mineral samples.

The Raman system was also utilized in detecting substances significant to security and defense applications. The system's capability to detect water and ice, the presence of water in various hydrous minerals, and various combustible chemicals was previously demonstrated. ${ }^{6}$

Figures 6 shows the Raman spectra of water and ice as measured by the Raman system. Strong Raman bands were observed at 3270 and $3440 \mathrm{~cm}^{-1}$ due to symmetric and antisymmetric stretching O-H vibrational modes of water molecules, respectively. In the measured Raman spectrum of ice, the location of the band is in the same region as that of liquid water, but it is distinctively characterized by a very sharp peak at $3140 \mathrm{~cm}^{-1}$. The formation of stronger hydrogen bond in ice causes the decrease in frequency of the symmetric $\mathrm{O}-\mathrm{H}$ stretching mode of water molecules. Therefore detection of Raman bands in this region does not only indicate presence of water; the shape of the spectrum can also be analyzed to distinguish between liquid water and ice.

Figure 7 shows the Raman spectra of hydrous minerals melanterite $\left(\mathrm{FeSO}_{4} \cdot 7 \mathrm{H}_{2} \mathrm{O}\right)$, epsomite $\left(\mathrm{MgSO}_{4} \cdot 7 \mathrm{H}_{2} 0\right)$, bischofite $\left(\mathrm{MgCl}_{2} \cdot 6 \mathrm{H}_{2} \mathrm{O}\right)$, and gypsum $\left(\mathrm{CaSO}_{4} \cdot 2 \mathrm{H}_{2} \mathrm{O}\right)$. These samples are dry and show no physical evidence of their water content. However, the measured Raman spectra of these hydrous minerals show Raman bands around $3400 \mathrm{~cm}^{-1}$, which, as previously stated, are due to the stretching modes of water molecules, indicating the presence of water in these minerals. Water molecules can exist in these minerals at temperatures significantly above the boiling point of water because the water molecules are chemically bonded with the minerals. Detection of water in hydrous minerals is therefore still possible even in dry environments which would otherwise show no sign of liquid water.

The measured Raman spectra of combustible substances, such as acetone and isopropanol are shown in Figure 8. Both substances show several bands in the low frequency region and a few bands in the high frequency region. It can be seen that the Raman bands of these substances are very prominent making them easily identifiable through Raman spectroscopy. 


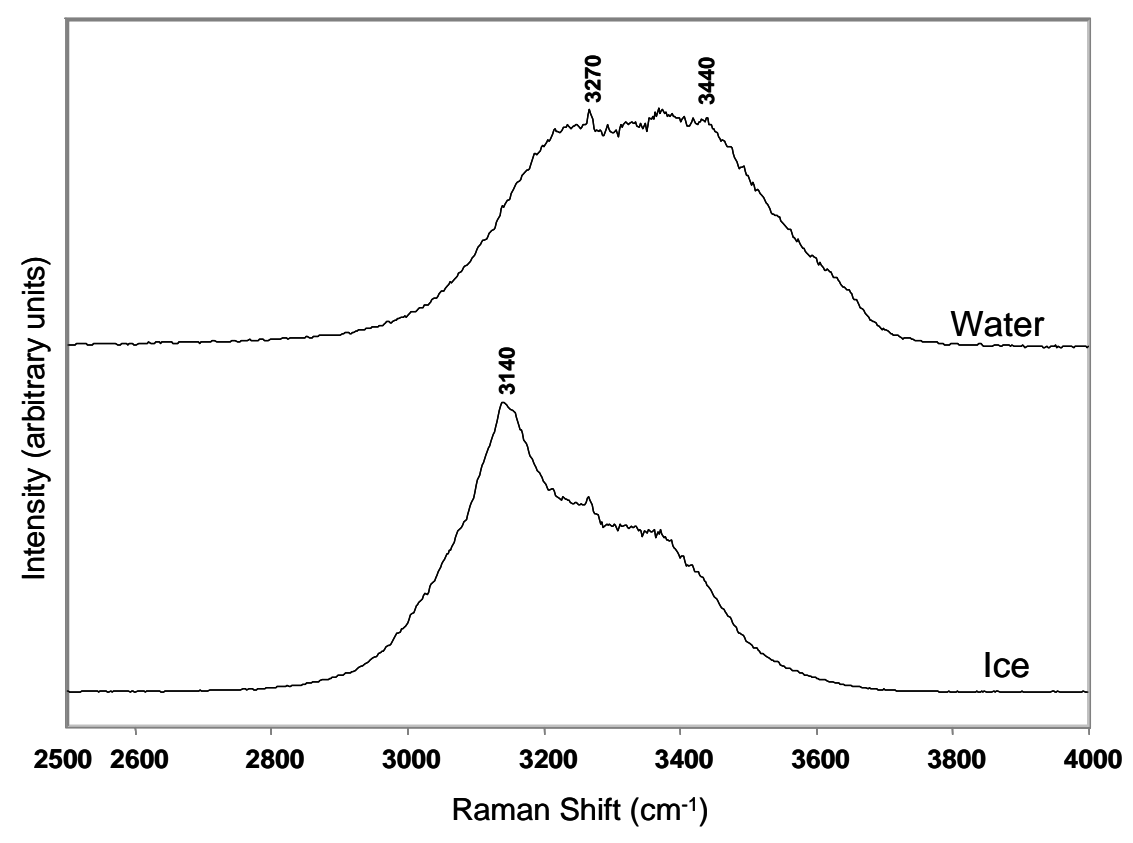

Figure 6. High frequency Raman spectra of liquid water and ice.

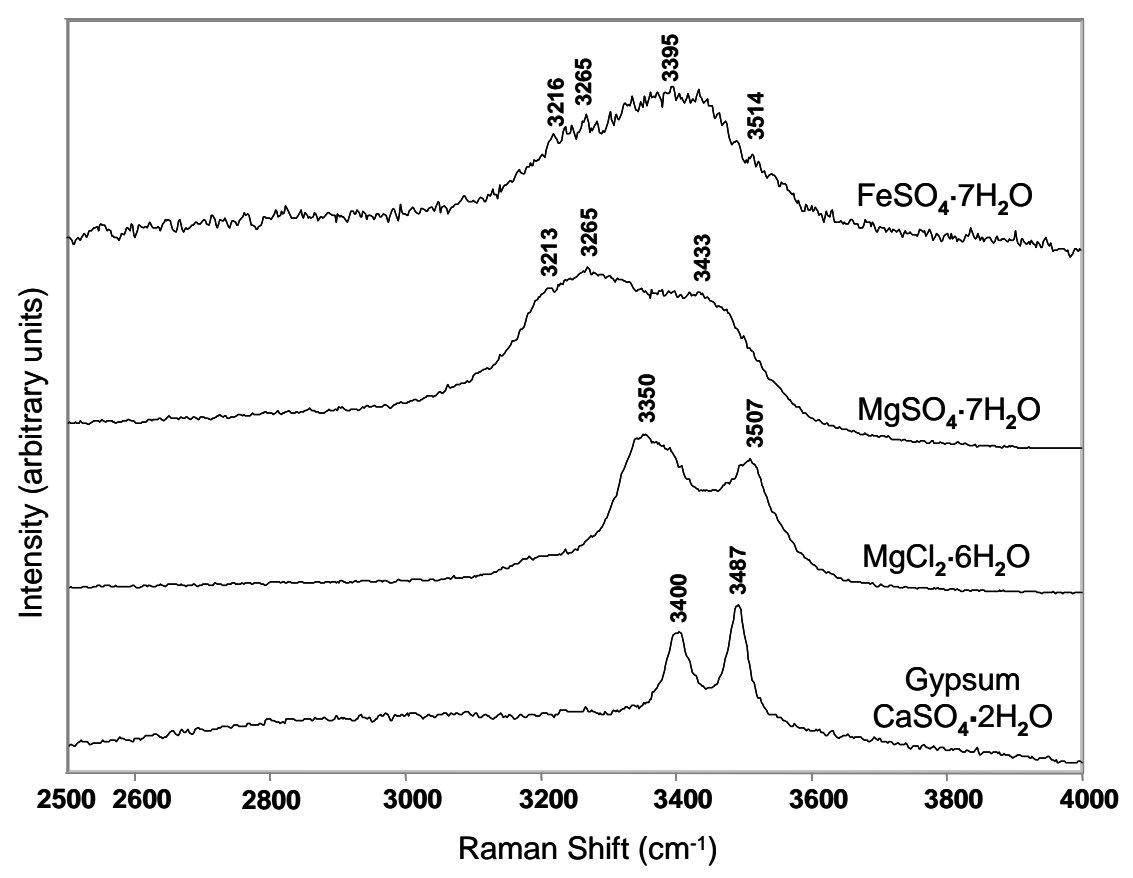

Figure 7. High frequency Raman spectra of hydrous minerals, $\mathrm{FeSO}_{4} \cdot 7 \mathrm{H}_{2} \mathrm{O}, \mathrm{MgSO}_{4} \cdot 7 \mathrm{H}_{2} \mathrm{O}, \mathrm{MgCl}_{2} \cdot 6 \mathrm{H}_{2} \mathrm{O}$, and gypsum. 


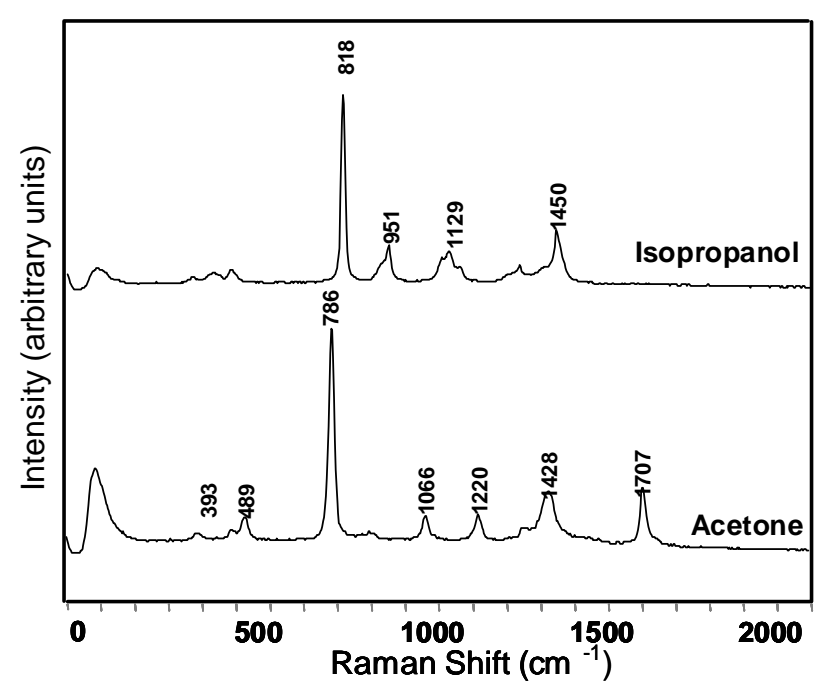

(a)

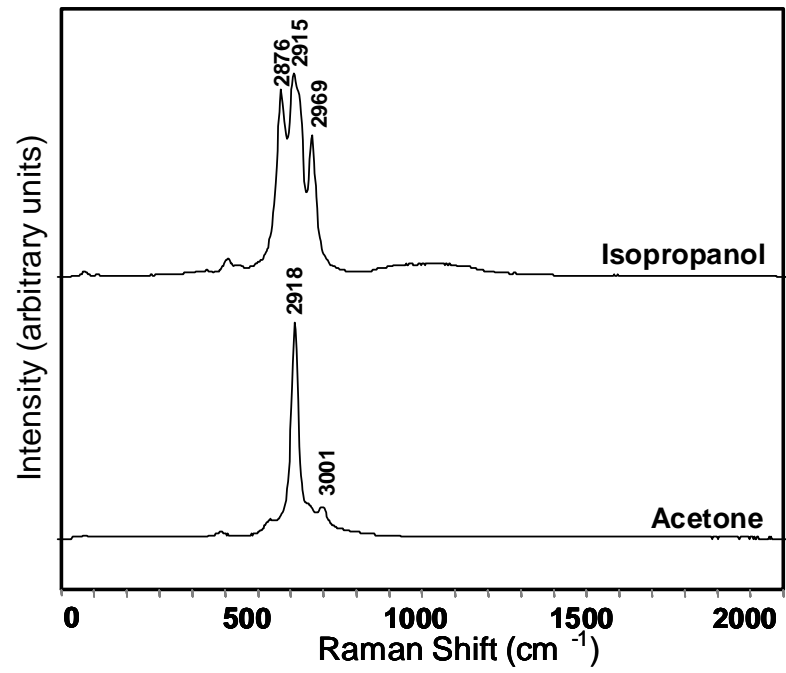

(b)

Figure 8. Raman spectra of isopropanol, acetone, naphthalene, and calcite in the (a) low frequency, and (b) high frequency shift regions

\section{CONCLUSION}

The Raman spectroscopy system demonstrated at NASA Langley Research Center successfully obtained the Raman spectra of various rocks and mineral samples under daylight conditions using a gated CCD detector. Minimizing the gate width of the CCD resulted in higher quality spectra with lower background and more prominent spectral peaks. The measured spectra of various rock samples were consistent with previously reported data. Furthermore, measurement of several samples of the same mineral yield consistent spectra with characteristic peaks in same location for each sample. Water and ice can be easily detected by the system, with the additional capability to distinguish between liquid water and ice. The presence of water can also be detected in hydrous minerals, which contain chemically bonded water molecules. Raman spectra of combustible substances such acetone and isopropanol were also obtained using the Raman system. Moreover, the Raman system's capability to detect substances that are significant to defense and security applications was also demonstrated.

\section{REFERENCES}

1. A. Wang, L. A. Haskin, A. L. Lane, T. J. Wdowiak, S. W. Squyres, R. J. Wilson, L. E. Howland, K. S. Manatt, N. Raouf, and C. D. Smith, “Development of Mars microbeam Raman Spectrometer,” J. Geophys. Res., Vol. 108, no. E1, 5005, 2003.

2. S. K. Sharma, S. M. Angel, M. Ghosh, H. W. Hubble, P. G. Lucey, "Remote pulsed Raman spectroscopy system for mineral analysis on planetary surfaces to 66 Meters,” Applied Spectroscopy,Vol. 56, 699-704, 2002.

3. A. K. Misra, S. K. Sharma, C. H. Chio, P. G. Lucey, B. Lienert, "Pulsed remote Raman system for daytime measurements of mineral spectra,” Spectrochim. Acta Part A, Vol. 61, 2281-2287, 2005.

4. J. D. Stopar, P. G. Lucey, S. K. Sharma, A. K. Misra, G. J. Taylor, H. W. Hubble, “ Raman efficiencies of natural rocs and minerals: Performance of a remote Raman system for planetary exploration at a distance of 10 meters," Spectrochim. Acta Part A, Vol. 61, 2315-2323, 2005.

5. S. K. Sharma, A. K. Misra, P. G. Lucey, S. M. Angel, C. P. McKay, “ Remote pulsed Raman spectroscopy system of Inorganic and organic materials to a radial distance of 100 meters,” Applied Spectroscopy, Vol. 60, no 8, 871876, 2006.

6. C. S. Garcia, M. N. Abedin, S. K. Sharma, A. K. Misra, S. Ismail, U. N. Singh, T. F. Refaat, H. E. Elsayed-Ali, S. P. Sandford, "Remote pulse laser Raman spectroscopy system for detecting water, ice, and hydrous minerals," 
Proceedings of SPIE, Vol. 6302, 2006.

7. S. M. Angel, T. J. Kulp, T. M. Vess, "Remote-Raman spectroscopy system at intermediate ranges using lowpower cw lasers" Applied Spectroscopy, Vol 46, no. 7, 1085-1091

8. Kaiser Optical Systems, Inc., "Holographic Notch Filters," Raman Products Technical Note No. 1050, http://www.kosi.com/raman/resources/technotes/1050.pdf.

9. Kaiser Optical Systems, Inc., "HoloPlex Holographic Transmission Grating,” Raman Products Technical Note No. 1201, http://www.kosi.com/raman/resources/technotes/1201.pdf.

10. Princeton Instruments, PI-MAX/PI-MAX2 System Manual, Version 5.B, p. 17, 2004.

11. NIST Standard Reference Database Website: http://webbook.nist.gov/chemistry.

12. W. D. Bischoff, S. K. Sharma, F. T. McKenzie, "Carbonate ion disorder in synthetic and biogenic magnesian calcites; a Raman spectral study,” Am. Mineral., Vol. 70, no. 5-6, 581-589, 1985.

13. S. D. Ross, in : V. C. Farmer (Ed), The Infrared Spectra of Minerals, Mineralogical Society, London, p 243, 1974.

14. W. F. Cole and C. J. Launcuki, "A refinement of crystal structure of gypsum $\mathrm{CaSO}_{4} \cdot 2 \mathrm{H}_{2} \mathrm{O}$," Acta Cryst. B30, 921, 1974.

15. C. H. Chio, S. K. Sharma, and D. W. Muenow, Raman spectroscopic studies of gypsum between 33 and 374 K, Amer. Mineral., 89, 390, 2004. 